\title{
A TOMADA DE DECISÃO APLICADAAO CUSTO TOTAL DE PROPRIEDADE EM UMA INSTITUIÇÃO DE ENSINO SUPERIOR PRIVADA
}

\author{
Julio Cezar Mairesse Siluk ${ }^{1}$ \\ Alvaro Luiz Neuenfeldt Júnior ${ }^{2}$ \\ Charlene Coser Dalcol ${ }^{3}$ \\ Marlon Soliman ${ }^{4}$
}

\begin{abstract}
Resumo: Atualmente,a utilização de ferramentas para a gestão finaneira em conjunto com metodologias capazes de apoiar à tomada de decisão visam possibilitar o esclarecimento sobre o papel dos diversos elementos presentes na negociação com os fornecedores, proporcionando a otimização dos recursos empregados para esse fim. Dessa forma, o artigo tem por objetivo apoiar a decisão no momento da compra de equipamentos de informáticaatravés da utilização dos conceitos a respeito do Custo Total de Propriedade e do Processo de Análise Hierárquica, para uma Instituição de Ensino Superior privada localizada na região central do Estado do Rio Grande do Sul, partindo inicialmente do caso da expansão de um de seus laboratórios de informática e da criação de uma nova sala para a modalidade de Ensino à Distância. Ao final do estudo, foi possível selecionar através dos critérios estipulados o fornecedor denominado por $f=1$, com $24 \%$ do resultado total, como o mais relevante em relação aos demais.
\end{abstract}

Palavras-Chave:Custo Total de Propriedade; Processo de Análise Hierárquica; Instituições de Ensino Superior; Competitividade.

\begin{abstract}
Currently, the use of tools for finance management in conjunction with methodologies capable of supporting the decision aim to provide clarification on the role of the different elements present in negotiating with suppliers, providing the optimization of the resources employed to that end. In this way, the article aims to support the decision at the time of the purchase computer equipment, through the use of the concepts regarding the Total Cost of Ownership and Analytic Hierarchy Proces, to a private higher education institution located in the central region of the Rio Grande do Sul state, starting initially in the case of the expansion of one computer labs and the creation of a new room for distance learning mode. At the end,it was possible to select, according to the criteria stipulated, that the supplier named by $f=1$, with $24 \%$ of the total result, as the more relevant in relation to the others.
\end{abstract}

Keywords:Total Cost of Ownership; Analytic Hierarchy Process;Higher Education Institution; Competitiveness.

\footnotetext{
${ }^{1}$ Universidade Federal de Santa Maria. Av. Roraima, 1000, prédio 7. Camobi. Santa Maria, RS. 97105-440. jsiluk@ufsm.br

${ }^{2}$ Universidade Federal de Santa Maria. Av. Roraima, 1000, prédio 7. Camobi. Santa Maria, RS. 97105-440. alvjr2002@hotmail.com

${ }^{3}$ Universidade Federal de Santa Maria. Av. Roraima, 1000, prédio 7. Camobi. Santa Maria, RS. 97105-440. charlenedalcol@gmail.com

${ }^{4}$ Universidade Federal de Santa Maria. Av. Roraima, 1000, prédio 7. Camobi. Santa Maria, RS. 97105-440. marlonsoliman@gmail.com
} 


\section{INTRODUÇÃO}

Em meio ao contexto dos anos 80, diversos questionamentos surgiram a respeito das formas com que a contabilidade gerencial era utilizada pelas organizações, tornando-se muitas vezes enganosas para os gestores e o público interessado, desfocando a atenção em relação aos verdadeiros fatores críticos que influenciam na manutenção e melhoria do desempenho empresarial (Kosier e Strong, 2006; Feiman 2009; Santos et al., 2010). Dentre os principais aspetos relacionados a essa obsolescência gerencial destaca-se a falta de operacionalidade das informações geradas, servindo apenas para divulgar a situação financeira, não envolvendo diretamente a redução dos custos e o aumento da produção industrial como um todo para auxiliar na tomada de decisão (Ramos, 2004; Degraeve et al., 2005; Lima Filho et al., 2011).

Em empresas onde o papel dos responsáveis pelas solicitações de compras para bens e serviços é considerado um elemento estratégico, uma das ferramentas que surgiram como evolução dessa forma paradigmática de pensamento é a chamada Custo Total de Propriedade (TCO), que se destaca por tratar a conceituação relativa aos custos sob a ótica da gestão do ciclo de vida de um produto, projeto ou serviço da empresa, a fim de se entender de maneira mais clara o papel dos diversos elementos das atividades envolvidas na negociação com os fornecedores, buscando otimizar o valor total investido no momento da escolha por uma das alternativas existentes no mercado (Zachariassen e Arlbjørn, 2011).

Porém, mesmo com a obtenção desses dados,é necessário que se busque outros métodos capazes de retornar aos decisores uma organização sistemática e coerente quantitativamente, a fim de apoiálos no momento de se realizar a determinação por um meio de fornecimento em detrimento aos outros, como é o caso encontrado para o Processo de Análise Hierárquica (AHP), considerado como um método capaz de atender a esse nível de exigência organizacional, devido as sua natureza intimamente ligada a verificação multicriterial existente entre as alternativas de um sistema (Saaty, 2008; Gomes e Gomes, 2012).

Logo, é proposto como objetivo do artigo apoiar a decisão no momento da compra de equipamentos de informáticaatravés da utilização dos conceitos a respeito do Custo Total de Propriedade e do Processo de Análise Hierárquica, para uma Instituição de Ensino Superior (IES) privada localizada na região central do Estado do Rio Grande do Sul, partindo inicialmente do caso piloto da expansão de um de seus laboratórios de informática e da criação de uma nova sala para a modalidade de Ensino à Distância (EAD).

A utilização de ambos os conceitos para esse tipo de pesquisa se justifica pela falta de controle atual daIESem relaçãoa atividade de compra desse tipo de equipamento, o que gera dispêndios financeiros extras, além de enfraquecer o nível de relacionamento existente com os fornecedores dos produtos e serviços.Ainda, Cousins et al. (2008) consideram em seus estudos que as atividades de compra relacionadas a bens podem representar, no mínimo, 50\% do custo total da empresa.Com esta informação, é possível afirmar que ao se propor esse tipo de aplicação na prática organizacional, por menores que sejam as reduções em custos conquistadas, há uma possibilidade de se conseguir impactar de maneira significativa os resultados operacionaisda empresa, sendo, portanto, uma área de alto potencial de interesse tanto no âmbito de estudos acadêmicocientíficos quanto do ambiente profissional.

Em relação ao público - alvo 
espera-se atingir com o estudo todos os envolvidos com esse tipo de atividade na IES, bem como de se tornar uma ferramenta que facilite a visão dos gestores a respeito da posição de cada uma das opções de fornecimento no mercado, tornando o processo de compra mais transparente como um todo. Academicamente, tem-se por pressuposto contribuir inovativamente por meio da união de dois conceitos considerados de naturezas distintas em prol de um mesmo objetivo, agregando para o desenvolvimento de futuros estudos a respeito de ambas as temáticas.

\section{CUSTO TOTAL DE PROPRIEDADE}

Através da necessidade cada vez maior de controle dos custos para se estimar mais precisamente o quanto um produto impacta na organização, o custeio total surgiu através de estudos realizados pelo GartnerGroup, empresa especializada em consultoria e pesquisas no ramo de TI, de maneira a mensurar quantitativamente $o$ desempenho das diversas formas de custos nas organizações, visando otimizar os recursos utilizados para o pleno funcionamento do sistema de trabalho (Degraeve et al., 2005).

Dentre as ferramentas inerentes ao custeio total, o Custo Total de Propriedade (TCO) foi definido como uma ferramenta focada nos custos de aquisição de bens para uma empresa, começando desde um alto nível de abstração dos dados até o nível de detalhamento, levando em conta o planejamento voltado para as tecnologias utilizadas até a implementação e administração dos recursos obtidos (Feiman, 2009).Saliba (2006) afirma que a correta análise do fluxo para o processo de compras através do TCO pode determinar de maneira clara quais formas de custo afetam mais significativamente os ganhos para o período estabelecido possibilitando, em longo prazo, a disponibilização de uma visão mais clara sobre qual a melhor opção existente no mercado.Com isso a modelagem tem, por definição, apurar ao máximo o custeio de um produto, serviço ou projeto durante um determinado tempo, de modo a gerenciar os custos como um todo, chegando ao que se considera como realidade da operação, que envolve a reformulação das estratégias adotadas em relação à negociação, cobrindo assim a demanda existente nas diversas etapas e situações encontradas para a compra de materiais (Ellram eZsidisin, 2002).

Dessa forma, o TCO exige mudanças na forma com que se trabalham as compras na empresa, através de uma mudança de filosofia e cultura de se realizar as rotinas, tornando realidade as expectativas geradas no momento das alterações propostas (Feiman, 2009). Para atenuar o efeito dessas modificações, é importante a realização do mapeamento dos processos de compras existentes, identificando os seus principais fatores inerentes, gerando um fluxo dessa operação determinado como ideal, perpetuando-se para operações futuras(Almeidaet al., 2010).

\section{INSTITUIÇÕES DE ENSINO SUPERIOR}

Com relação à temática no Brasil, essa modalidade empresarial pode ser definida a partir de organizações prestadoras de serviços que contemplam as atividades de ensino, pesquisa e extensão, de maneira a contribuir para a formação educacional de jovens e adultos para o exercício profissional, denotando um sistema complexo, diversificado e em constante expansão, sendo classificadas, segundo o Ministério da Educação do Brasil, em públicas (federais, estaduais e municipais) e privadas (comunitárias,confessionais, filantrópicas e particulares).

Para Hansen (2001), as Instituições de Ensino Superior (IES) ainda relutam em enfrentar seu posicionamento na sociedade como empresas, de modo que algumas têm 
dificuldade de se reconhecer como organizações empresariais, não utilzando de quaisquer modelos de administração para a sua gestão.Em relação à administração dos recursos de compras, Merlo e Beuren (2004) citam que a gestão econômica e financeira de uma instituição de ensino é considerada como atividademeio, por estabelecer um suporte as atividades que realmente agregam e representam os objetivos da organização, mas que independente dessa classificação, são elencadas como de alta necessidade e importância, principalmente quando vinculadas a compra de materiais e a solicitação de serviços.

Progressos nessas áreas de gestão vêm sendo notados a partir dos anos $90 \mathrm{em}$ IES norte-americanas, através de relativas evoluções nas formas com que os custeios eram realizados, adotando-se de técnicas capazes de clarificar a forma com que a alocação dos recursos estava relacionada aos conceitos de custo dos produtos e serviços contratados. No Brasil, conforme descrito por Menegat (2006), atualmente essas sistemáticas de trabalho são amplamente utilizadas como base no auxílio para a tomada de decisões, faciltando de maneira direta em sua gestão como um todo,permeando desde a correta precificação dos produtos ofertados aos clientesaté o acompanhamento economicofinanceiro para os mais diversos projetos existentes.

\section{O PROCESSO DE ANÁLISE HIERÁRQUICA}

A fim de conduzir para a escolha das melhores alternativas em relação aos cenários de desenvolvimento reconhecidos como de complexidade, os métodos multicritérios tem por premissa básica auxiliar os decisores no momento dos julgamentos das alternativas a respeito das decisões factíveis e não factíveis propostas a um problema em questão, havendo a necessidade, para tanto, de critérios (ou fatores) nos quais determinam as características consideradas como predominantes do sistema (Gomes e Gomes, 2012; Saaty e Vargas, 2012).

Dentre as diversas abordagens existentes para o apoio multicritério, o Processo de Análise Hierárquica (AHP) é considerado como um modelo capaz de compreender matematicamente as características para a decisão entre duas ou mais alternativas, de modo a se propor o julgamento da importância relativa de cada um delas através de um comparativo par a par, por meio de uma estrutura hierárquica onde cada um deles se localizará conforme o seu nível de relação (Saaty, 2008; Gomeset al., 2011). Dessa forma, pode-se afirmar que os métodos AHP mais consagrados por sua aplicação prática são o AHP Clássico (Saaty, 1980), o AHP Multiplicativo (Lootsma, 1993), o AHP Referenciado (Watson e Freeling, 1982), o AHP B-G (Belton e Gear, 1985), o AHP com Nebulosidade, o AHP com Interpretação Probabilística e o Processo de Análise em Redes (ANP) (Saaty, 1980), sendo a escolha por algum destes definidos prioritariamente conforme as características demonstradas pela situação ao qual se deseja verificar.

Em específico, Gomes et al. (2011) citam que os Processos de Análise Hierárquica Referenciada e B-G surgiram como uma evolução natural da abordagem clássica proposta por Saaty (1980), onde o primeiro exprime a preocupação com a controvérsia surgida a partir da relação pressuposta entre os valores dos critérios em relação às alternativas, introduzindo-se para tanto uma constante de proporcionalidade $K$ para a comparação entre elas, enquanto para o segundo está baseado na inversão do ordenamento das alternativas do sistema em relação à introdução de mais uma no sistema, buscando-se para mitigar esse problema através da determinação de um valor $z_{\max }$ capaz de referenciar a alternativa com maior resultado relativo, a fim de se realizar a 
normalização dos resultados absolutos encontrados (Watson e Freeling, 1982; Belton e Gear, 1985).

Para tanto, ambos devem considerar em sua constituição fatores $(d)$ elencados, os quais influenciam de maneira direta na seleção das alternativas $(f)$ propostas, $\forall d \in\{1,2, \ldots, n\} \quad$ e $\quad \forall f \in\{1,2, \ldots, n\}$. Em relação ao método Referenciado, conforme descrito anteriormente, é necessário se determinar primeiramente a constante proporcional $K$ a ser adotada, de acordo com as características das unidades de medida do modelo, seguido dos fatores denominados por escala $\left(q_{d}\right)$ que definem a medida de cada critério proporcionalmente as unidades do objetivo, para se chegar, finalmente, ao cálculo da importância de cada um deles $\left(x_{d}\right)$, conforme descrito pela Equação (1), baseado nos pressupostos descritos por Gomes et al. (2011),

$$
\mathrm{x}_{\mathrm{d}}=\mathrm{K} * \mathrm{q}_{\mathrm{d}} * \sum_{\mathrm{d}=1}^{\mathrm{n}}\left(\mathrm{T}_{\mathrm{f}}\right)_{\mathrm{d}}
$$

onde $T_{f}$ (ou $T_{f}$ ) é o módulo relativo ao valor absoluto do fator em relação à alternativa estudada no momento. Quanto ao grau de priorização de cada alternativa em relação aos fatores elencados, é proposta a elaboração das matrizes $C_{d_{n * n}}=\left(c_{f f^{\prime}}\right)$ baseadaem Saaty (2008),tal que o número de julgamentos necessários para a construção de cada uma é definido através da relação $n^{*}(n-1) / 2$, de maneira que $n$, nesta situação, compreende $\mathrm{o}$ número total de elementos $c_{f f^{\prime}}$ existentes na matriz. Com isso, devem ser atribuídos para cada elemento os valores encontrados para as alternativas $\left(T_{f}\right)$ em relação ao fator $d$ determinante da Matriz (3), caracterizando quantitativamente a relação de importância entre eles, de acordo com a Equação (2).

$$
\mathrm{c}_{\mathrm{ff}}=\frac{\mathrm{T}_{\mathrm{f}}}{\mathrm{T}_{\mathrm{f}^{\prime}}}\left\{\begin{array}{l}
\forall \mathrm{f}=(1,2, \ldots, \mathrm{n}) \\
\forall \mathrm{f}^{\prime}=(1,2, \ldots, \mathrm{n})
\end{array}\right.
$$

$$
\mathrm{C}_{\mathrm{d}_{\mathrm{n} * \mathrm{n}}}=\left[\begin{array}{cccc}
\mathrm{T}_{1} / \mathrm{T}_{1} & \mathrm{~T}_{1} / \mathrm{T}_{2} & \cdots & \mathrm{T}_{1} / \mathrm{T}_{\mathrm{n}} \\
\mathrm{T}_{2} / \mathrm{T}_{1} & \mathrm{~T}_{2} / \mathrm{T}_{2} & \cdots & \mathrm{T}_{2} / \mathrm{T}_{\mathrm{n}} \\
\vdots & \vdots & \ddots & \vdots \\
\mathrm{T}_{\mathrm{n}} / \mathrm{T}_{1} & \mathrm{~T}_{\mathrm{n}} / \mathrm{T}_{2} & \cdots & \mathrm{T}_{\mathrm{n}} / \mathrm{T}_{\mathrm{n}}
\end{array}\right]
$$

Logo, por meio dessa matriz de decisão é possível o cálculo dos vetores para os resultados do seu conjunto de fatores constituintes $\quad\left(\bar{v}_{f}\left(C_{d_{n * n}}\right)\right.$ ), conforme a Equação (4), normalizados por intermédio do vetor $\bar{v}_{P_{f}}\left(C_{d_{n * n}}\right)$ em relação aos resultados obtidos para as alternativas, de acordo com a Equação (5), baseando-se nos pressupostos de Gomes et al. (2011),

$$
\begin{gathered}
\overline{\mathrm{v}}_{\mathrm{f}}\left(\mathrm{C}_{\mathrm{d}_{\mathrm{n} * \mathrm{n}}}\right)=\sum_{\mathrm{f}^{\prime}=1}^{\mathrm{n}} \mathrm{c}_{\mathrm{ff}} \\
\overline{\mathrm{v}}_{\mathrm{P}_{\mathrm{f}}}\left(\mathrm{C}_{\mathrm{d}_{\mathrm{n} * \mathrm{n}}}\right)=\frac{\overline{\mathrm{v}}_{\mathrm{f}}\left(\mathrm{C}_{\mathrm{d}_{\mathrm{n} * \mathrm{n}}}\right)}{\sum_{\mathrm{f}=1}^{\mathrm{n}} \overline{\mathrm{v}}_{\mathrm{f}}\left(\mathrm{C}_{\mathrm{d}_{\mathrm{n} * \mathrm{n}}}\right)}
\end{gathered}
$$

de maneira que o $\sum_{f=1}^{n} \bar{v}_{f}\left(C_{d_{n * n}}\right)=1$, além da importância relativa entre as alternativas $\left(w_{f}\right)$ observado para o Processo de Análise Hierárquica Referenciada através da relação entre o valor encontrado em $x_{d}$ e o vetor de normalização da matriz $\bar{v}_{P_{f}}\left(C_{d_{n * n}}\right)$ de cada uma delas, resultando na proposta descrita pela Equação (6):

$$
\mathrm{w}_{\mathrm{f}}=\sum_{\mathrm{d}=1}^{\mathrm{n}} \mathrm{x}_{\mathrm{d}} * \overline{\mathrm{v}}_{\mathrm{P}_{\mathrm{f}}}\left(\mathrm{C}_{\mathrm{d}_{\mathrm{n} * \mathrm{n}}}\right)
$$

Para o processo de análise $\mathrm{B}-\mathrm{G}$ primeiramente deve-se encontrar, dentro de cada matriz $C_{d_{n * n}}$, o maior valor $\operatorname{de} \bar{v}_{f}\left(C_{d_{n * n}}\right)$, denominado por $\mathbf{z}_{\text {max }}$, a fim de tornar este proporcional a uma unidade de medida em relação aos outros valores da matriz $C_{d_{n * n}}$, de acordo com o procedimento estabelecido pela Equação (7),

$$
\max _{\mathrm{f} \in \mathrm{C}_{\mathrm{d}_{\mathrm{n} * \mathrm{n}}}} \overline{\mathrm{v}}_{\mathrm{f}}\left(\mathrm{C}_{\mathrm{d}_{\mathrm{n} * \mathrm{n}}}\right)=\mathrm{z}_{\max } \propto 1 ; \text { sujeito }
$$




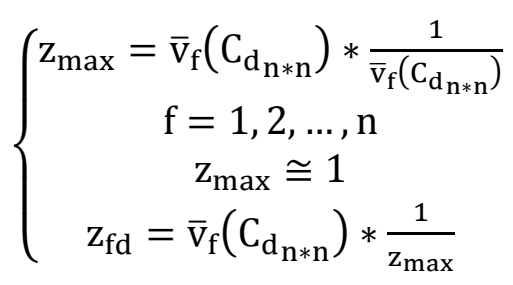

obtendo-se como produto os elementos $Z_{f d}$ proporcionais ao valor máximo que estarão dispostos na matriz de julgamentos $A_{n * n}$, sendo o valor da importância de cada fator $\left(\delta_{d}\right)$ proposto pela Equação (8) e, consequentemente, o cálculo pela Equação (9) para cada alternativa estabelecida $\left(\mu_{f}\right)$.

$$
\begin{aligned}
\delta_{\mathrm{d}} & =\mathrm{K} * \mathrm{q}_{\mathrm{d}} * \mathrm{z}_{\max } \\
\mu_{\mathrm{f}} & =\sum_{\mathrm{d}=1}^{\mathrm{n}} \delta_{\mathrm{d}} * \mathrm{z}_{\mathrm{fd}}
\end{aligned}
$$

Por fim, com os resultados estabelecidos pelos dois métodos é possível se obter a média dos valores $\left(\rho_{f}\right)$, nos quais se estabelecerá a pré-ordem entre as alternativas selecionadas, bem como da diferença entre eles $\left(\Delta_{f}\right)$, a fim de verificar o nível de discrepância para o caso, conforme as Equações (10) e (11),

$$
\begin{gathered}
\rho_{\mathrm{f}}=\frac{\left(\mu_{\mathrm{f}}+\mathrm{w}_{\mathrm{f}}\right)}{\mathrm{p}} \\
\Delta_{\mathrm{f}}=\mu_{\mathrm{f}}-\mathrm{w}_{\mathrm{f}}
\end{gathered}
$$

ondep é proporcional ao número de métodos adotados para a verificação.

\section{METODOLOGIA}

A presente pesquisa está composta metodologicamente por meio de uma estrutura composta pordozeetapas distintas, observadas desde a contextualização teórica dos temas abordados até as conclusões com a aplicação do TCO, conforme mostra a Figura 1.

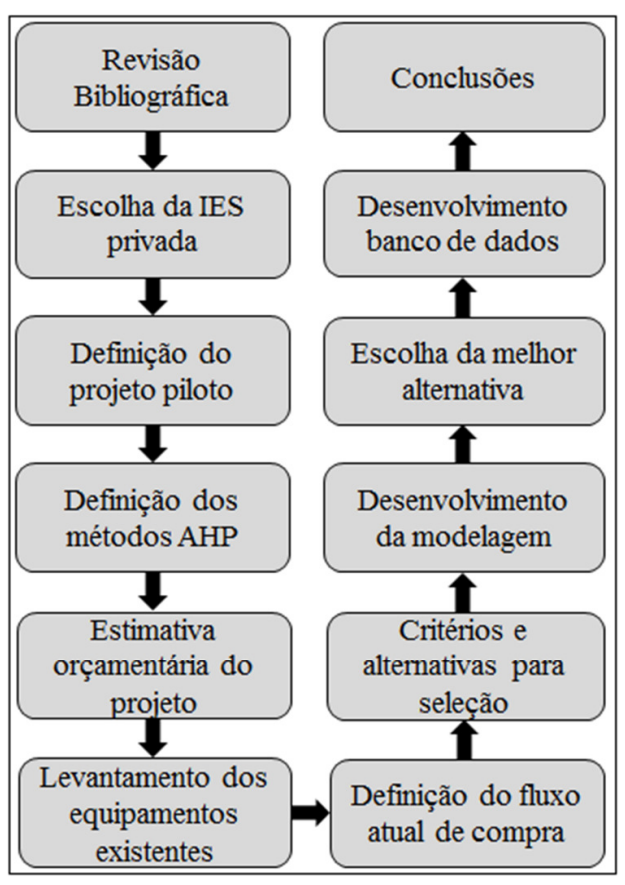

Figura 1 - Etapas do desenvolvimento do modelo.

Como base para certificação a respeito das técnicas e ferramentas utilizadas no estudo, foi proposta inicialmente a pesquisa bibliográfica, utilizando-se prioritariamente o portal de periódicos da Coordenação de Aperfeiçoamento de Pessoal de Nível Superior (CAPES), assim como os portais Scientific Direct, Emerald e Scopus, a fim de se obter artigos publicados em revistas tanto relacionadas ao âmbito nacional quanto internacional. Além disso, foram pesquisadas referências em livros sobre os principais autores e anais de congressos.

Como referência para o estudo, escolheu-se uma IES localizada na região central do estado do Rio Grande do Suldevido à facilidade de acesso aosdados necessários. Em específico, definiu-se como projeto piloto o estudo a respeito da expansão de um de seus laboratórios de informática e da criação de uma nova sala para a modalidade EAD, demandas de curto prazo vigentes para a atual administração da empresa.

Quanto da definição dos métodos aplicados ao conjunto de dadosescolheu-se 
o AHP Referenciada e a B-G conjuntamente,com base na natureza dos dados a respeito da formatação do TCO da empresa e nos pressupostos descritos por Guitouni e Martel (1998) para se elencar um modelo multicritério que melhor compreenda as características do sistema, partindo-se inicialmente da definiçãodas alternativas e critérios aos quais os julgamentos serão estabelecidos, para se obter ao final a que define melhor a necessidade de compra para o sistema.

Com isso tornou-se possívelo levantamento dos dados a respeito da empresa, a partir de um diagnóstico semiestruturado submetido aos funcionários e gestores de Tecnologia da Informação (TI) e o corpo diretivo da empresa, obtendo-se assim os atuais equipamentos existentes, o orçamento estipulado para a compra, o fluxo das informações e a estimativa econômica da proposta considerada como ideal para o projeto, partindo assim para a definição dos três critérios (qualitativo, quantitativo e econômico) considerados como básicos para se realizar a seleção, de acordo com os pressupostos descritos por Drucker e Marcianello (2005), Lobato et al. (2006), Saliba (2006), Van der Stede et al. (2006), Kaplan e Norton (2008), Bandeira (2009) e Porter (2009), gerando por consequência os indicadores capazes de retornar a mensuração das cinco alternativas de fornecedores,elencadas em relação ao mercado local e regional, por meio de pesquisas através de sites, guias telefônicos, histórico de compras e indicação de pessoas da área de TI.

A fim de atender a demanda por métricas quantitativas capazes de representar o comportamento de cada um dos critérios estabelecidos anteriormente, tem-se a proposta a etapa de desenvolvimento da modelagem matemática para avaliação do problema, no qual demanda, para fins de julgamento, da definição de um fator de escala padrão (Likert), possibilitando a normalizaçãoda verificação dos resultados obtidos, facilitando a comparação dos indicadores por meio da conversão desta para uma unidade de medida padrão.

Por fim, ocorre a escolha propriamente dita da melhor alternativa proposta em relação ao sistema,consolidados em um banco de dados organizado com base nas informações obtidas com a aplicação do projeto piloto.

\section{APLICAÇÃO E RESULTADOS OBTIDOS}

Inicialmente consultou-se o corpo diretivoda IES para a verificação do valor disponibilizado para a compra dos equipamentos necessários para o projeto, bem como das características dos laboratórios, definidos neste estudo pela denominação SALAS, onde se chegou a configuração de dois locais, um definido para ser a expansão do laboratório informatizado para pesquisas técnicocientíficas dos discentes, passando de uma capacidade atual de 20 para 40 lugares (SALA 1), e outro, sem qualquer estrutura de TI previamente existente, capaz de atender a futuras aulas a serem ministradas sob a metodologia de Ensino à Distância, com um número de 30 usuários simultaneamente $(+1$ para 0 instrutor $)$ (SALA 2). Para tanto, a IES disponibilizou uma verba total de $\mathrm{R} \$ 100.000,00$, destinada exclusivamente a compra de equipamentos de informática.

Com isso, a fim de entender melhor o sistema e de verificar economicamente a relação de compra referentea abertura e ampliação dessas duas salas informatizadas, inicialmente se propõe o estudo dos atuais equipamentos existentes na SALA 1, no qual estão elencados de acordo com os seus preços estimados para o mercado atual, conforme mostra a Tabela 1. 
Tabela 1 - Equipamentos atualmente existentes

\begin{tabular}{|c|c|c|c|}
\hline \multicolumn{4}{|c|}{ EQUIPAMENTOS ATUAIS } \\
\hline Descrição & Qde. & $\begin{array}{c}\text { Preço Un. } \\
(\mathbf{R} \$)\end{array}$ & $\begin{array}{c}\text { Preço Total } \\
\text { (R\$) }\end{array}$ \\
\hline Desktops & 20 & 600,00 & $12.000,00$ \\
\hline Monitores & 20 & 200,00 & $4.000,00$ \\
\hline $\begin{array}{c}\text { Servidor para } \\
\text { aplicação dados }\end{array}$ & 1 & 950,00 & 950,00 \\
\hline Switch & 1 & 150,00 & 150,00 \\
\hline Nobreak & 4 & 250,00 & $1.000,00$ \\
\hline Storage System & 1 & 800,00 & 800,00 \\
\hline \multicolumn{3}{|c|}{ Total } & $\mathbf{1 8 . 9 0 0 , 0 0}$ \\
\hline
\end{tabular}

É possível comprovar que o total existente em equipamentos para a SALA 1, em valores atuais, está estimado em aproximadamente R\$ 18.900,00, sendo importante ressaltar que estes se encontram em perfeito estado e possuem condições de serem reaproveitados, mesmo que de alguma forma possam ser considerados como obsoletos para o mercado. Consolidados esses valores foi possível se formatar, em conjunto com os profissionais de TI e com a aprovação da diretoria da empresa, a proposta de compra considerada como de nível básico tecnicamente para se atender satisfatoriamente as exigências operacionais dos laboratórios, partindo-se das modalidades de equipamentos e dos valores descritos a partir das Tabelas 2 e 3 .

Nota-se que diferentemente da situação atual uma nova categoria está indexada ao levantamento, denominada por Thin Clients(consequentemente um servidor para esta função), que podem ser considerados como computadores clientes, através da sua relação direta com um servidor,sendo de uso prioritário para o processamento de informações a partir da internet, estando, portanto, de acordo com as necessidades de utilização requisitadas para as salas propostas, apesar do seu nível reduzido de processamento em relação aos Desktops.
Tabela 2 - Estimativa de matérias e orçamento da Sala 1

\begin{tabular}{|c|c|c|c|}
\hline \multicolumn{4}{|c|}{ SALA 1} \\
\hline Descrição & Qde. & $\begin{array}{l}\text { Preço Un. } \\
\text { (R\$) }\end{array}$ & $\begin{array}{c}\text { Preço Total } \\
(\mathbf{R} \$)\end{array}$ \\
\hline $\begin{array}{c}\text { Desktop } \\
\text { (existentes) }\end{array}$ & 20 & 600,00 & $12.000,00$ \\
\hline Thin Client & 20 & 480,00 & $9.600,00$ \\
\hline $\begin{array}{l}\text { Monitores } \\
\text { (existentes) }\end{array}$ & 20 & 200,00 & $4.000,00$ \\
\hline Monitores (novos) & 20 & 300,00 & $6.000,00$ \\
\hline $\begin{array}{c}\text { Servidor para } \\
\text { aplicação de } \\
\text { dados (existente) }\end{array}$ & 1 & 950,00 & 950,00 \\
\hline $\begin{array}{l}\text { Servidor para } \\
\text { aplicação de } \\
\text { dados (novo) }\end{array}$ & - & - & - \\
\hline $\begin{array}{c}\text { Servidor para } \\
\text { Thin Client }\end{array}$ & 1 & $2.900,00$ & $2.900,00$ \\
\hline Switch (existente) & 1 & 150,00 & 150,00 \\
\hline Switch (novo) & 1 & 250,00 & 250,00 \\
\hline $\begin{array}{c}\text { Nobreak } \\
\text { (existentes) }\end{array}$ & 4 & 250,00 & $1.000,00$ \\
\hline Nobreak (novos) & 4 & 500,00 & $2.000,00$ \\
\hline Storage (existente) & 1 & 800,00 & 800,00 \\
\hline Storage (novo) & - & - & - \\
\hline $\begin{array}{c}\text { Cabos, } \\
\text { Conectores, etc. }\end{array}$ & 1 & 350,00 & 350,00 \\
\hline $\begin{array}{l}\text { (+) Total } \\
\text { Proposta }\end{array}$ & & & $40.000,00$ \\
\hline $\begin{array}{c}\text { (-) Total } \\
\text { Reutilizado }\end{array}$ & & & $18.900,00$ \\
\hline $\begin{array}{c}\text { (=) Total } \\
\text { Investimentos }\end{array}$ & & & $21.100,00$ \\
\hline
\end{tabular}

Além disso, houve uma demanda extra pela compra de cabos, conectores e outros equipamentos para a instalação dos equipamentos novos em ambos locais. De acordo com a proposta é possível observar que esta demanda um custo total do projeto estimado em R\$78.230,00, sendo $24 \%$ desse valor relativo aos atuais equipamentos existentes na SALA 1 e o restante, $76 \%(\mathrm{R} \$ 59.330,00)$ ao custo básico $(C B)$ para a compra de novos, compondo assim a estimativa estrutural e orçamentária básica de estudo das alternativas e do TCO de compra dos equipamentos. 
Tabela 3 - Estimativa de matérias e orçamento da Sala 2

\begin{tabular}{|c|c|c|c|}
\hline \multicolumn{4}{|c|}{ SALA 2 } \\
\hline Descrição & Qde. & $\begin{array}{c}\text { Preço } \\
\text { Un. (R\$) }\end{array}$ & $\begin{array}{c}\text { Preço Total } \\
\text { (R\$) }\end{array}$ \\
\hline $\begin{array}{c}\text { Desktop } \\
\text { (existentes) }\end{array}$ & - & - & - \\
\hline Thin Client & 31 & 480,00 & $14.880,00$ \\
\hline $\begin{array}{c}\text { Monitores } \\
\text { (existentes) }\end{array}$ & - & - & - \\
\hline Monitores (novos) & 31 & 300,00 & $9.300,00$ \\
\hline $\begin{array}{c}\text { Servidor para } \\
\text { aplicação de dados } \\
\text { (existente) }\end{array}$ & - & - & - \\
\hline $\begin{array}{c}\text { Servidor para } \\
\text { aplicação de dados } \\
\text { (novo) }\end{array}$ & 1 & $2.900,00$ & $2.900,00$ \\
\hline $\begin{array}{c}\text { Servidor para Thin } \\
\text { Client }\end{array}$ & 2 & $2.900,00$ & $5.800,00$ \\
\hline Switch (existente) & - & - & - \\
\hline Switch (novo) & 2 & 250,00 & 500,00 \\
\hline $\begin{array}{c}\text { Nobreak } \\
\text { (existentes) }\end{array}$ & - & - & - \\
\hline Nobreak (novos) & 6 & 500,00 & $3.000,00$ \\
\hline Storage (existente) & - & - & - \\
\hline Storage (novo) & 1 & $1.500,00$ & $1.500,00$ \\
\hline $\begin{array}{c}\text { Cabos, Conectores, } \\
\text { etc. }\end{array}$ & 1 & 350,00 & 350,00 \\
\hline $\begin{array}{c}\text { (+) Total } \\
\text { Proposta }\end{array}$ & $\begin{array}{c}\text { (-) Total } \\
\text { Reutilizado }\end{array}$ & $\mathbf{3 8 . 2 3 0 , 0 0}$ \\
\hline $\begin{array}{c}\text { (=) Total } \\
\text { Investimentos }\end{array}$ & - & $\mathbf{3 8 . 2 3 0 , 0 0}$ \\
\hline
\end{tabular}

Quanto às atuais etapas inerentes ao processo de compra, não há um procedimento operacional padrão estabelecido para as solicitações oriundas da área de TI, sendo proposto inicialmente o estudo da maneira com que essa tarefa é seguida habitualmente pelo atual corpo de colaboradores da empresa, de acordo com o exposto pela Figura 2.

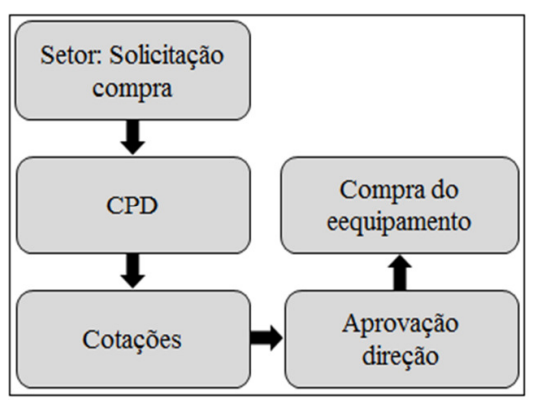

Figura 2 - Fluxo de compra atual para os equipamentos de TI

A compra dos equipamentos inicia com a solicitação, por parte do interessado, ao setor de processamentos de dados da IES (CPD), onde este por sua vez realiza três cotações simples de orçamentos com empresas escolhidas de maneira aleatória, obtendoapós a escolha por um destes um documento a ser submetido para a aprovação do corpo diretivo da organização que, por fim, é enviada para o fornecedor. Nota-se que em nenhum momento está atribuído as questões que tratam o relacionamento com o fornecedor, tão pouco do controle das últimas compras realizadas pela empresa, flexibilidade e condições de negociação, não havendo um controle dos equipamentos adquiridos, do seu uso ou do nível de obsolescência atual.

Com base nesses fatos, na organização dos dados a partir do TCO realizadodurante $\mathrm{o}$ projeto piloto e nas estruturas bibliográficas apresentadas pelos autores referenciados, foi possível se elencar os fatores $(C d)$ aos quais devem guiar a empresa nesta e nas outras escolhas a serem consolidadas para esse tipo decompra, partindo de uma verificação que contempla um total de oito indicadores $I u$, $\forall u \in\{1,2, \ldots, 8\}$, conforme mostra a proposta descrita pela Figura 3. 


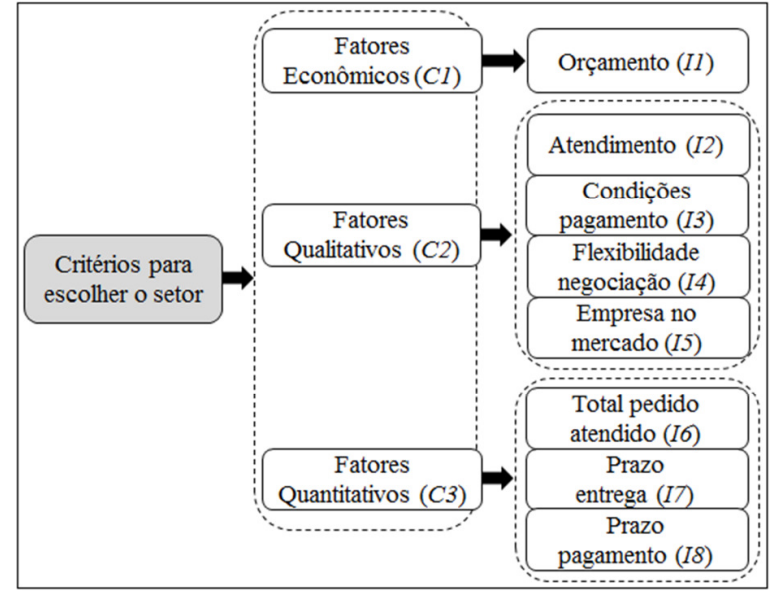

Figura 3 - Estrutura de verificação para a proposta

É possível perceber que destes apenas o primeiro pertence a questões exclusivamente econômicas, quatro são de cunho fortemente relacionado aquestão qualitativa e três de natureza quantitativa, aos quais foram submetidos para a verificação das cinco empresas elencadas como alternativas $(f)$ viáveis de se comprar os equipamentos necessários, $\forall f \in$ $\{1,2, \ldots, 5\}$. Porém, antes de realizar a escolha, deve-se definir as formas de se mensurar numericamente cada $I u$, de modo que estes servem como inputs para a consolidação dos modelos AHP determinados anteriormente. Para tanto, a fim de facilitar a verificação do sistema, buscou-se elencar todos $I u_{f}$ por meio de uma unidade de medida padronizada baseada na escala Likert, que contempla uma faixa de valores separada em cinco escalas distintas, denominada como $\alpha$, partindo desde um mínimo definido como 1 até um máximo equivalente a 5 , passando pelos valores 2, 3 (intermediário) e 4 .

Primeiramente, o cálculo do orçamento $\left(I 1_{f}\right)$ está proposto de forma a contemplar uma comparação entre o custo básico $(C B)$ estimado para a solicitação dos materiais relacionados no projeto e o custo orcamentário $\left(\mathrm{CO}_{f}\right)$ obtido por meio da consulta de cada um dos fornecedores elencados para a verificação, resultando como produto $\mathrm{o}$ índice orçamentário $\beta_{f}$ proposto pela Equação (12), que por intermédio da comparação com os valores pressupostos pela escala $\alpha$ torna possível a determinaçãode $I 1_{f}$, conforme mostra a Equação (13),

$$
\begin{gathered}
\beta_{\mathrm{f}}=\frac{\mathrm{CO}_{\mathrm{f}}}{\mathrm{CB}}-1 \\
\mathrm{I} 1_{\mathrm{f}}=\left\{\begin{array}{c}
1 \rightarrow \beta_{\mathrm{f}} \geq 0,06 \\
2 \rightarrow 0,031<\beta_{\mathrm{f}}<0,06 \\
3 \rightarrow-0,031 \leq \beta_{\mathrm{f}} \leq 0,0031 \\
4 \rightarrow-0,031<\beta_{\mathrm{f}}<-0,06 \\
5 \rightarrow \beta_{\mathrm{f}} \geq-0,06
\end{array}\right.
\end{gathered}
$$

onde é possível observar que quanto maior o valor de $C O_{f}$ em relação à $C B$, menor será o valor proporcional do indicador $I 1_{f}$ para a alternativa avaliada no momento, entendendo que quanto mais reduzido for o montante da proposta melhor será o resultado para a fornecedora. Quanto aos valores parametrizados para a comparação de $\beta_{f}$, estes foram determinados com base nas expectativas dos gestores da empresa para com os resultados.

$\mathrm{O}$ indicador $I 2_{f}$, denominado por atendimento, compreendeos fatores relacionados a maneira com que o contato se estabeleceu entre ambas as partes do negócio, partindo de uma análise qualitativa através do diagnóstico semiestruturado submetido aos envolvidos $p, \forall p \in\{1,2, \ldots, n\}$, das áreas envolvidas $s, \forall s \in\{1,2, \ldots, n\}$, no caso compras e TI e por projeto em questão $o$, $\forall o \in\{1,2, \ldots, n\}$. Os fornecedores são avaliados por meio da base numérica compreendida no fator escala $\alpha$, gerando por consequência o valor designado pelo índice $S O_{p}$,gerando assim a definição quantitativa para a mensuração $\operatorname{de} I 2_{f}$, conforme mostra aEquação (14):

$$
\mathrm{I} 2_{\mathrm{f}}=\sum_{\mathrm{o}=1}^{\mathrm{n}} \sum_{\mathrm{s}=1}^{\mathrm{n}} \sum_{\mathrm{p}=1}^{\mathrm{n}} \frac{\left[\frac{\left(\frac{\mathrm{SO}}{\mathrm{n}}\right)_{\mathrm{s}}}{\mathrm{n}}\right]_{\mathrm{o}}}{\mathrm{n}}
$$


De maneira semelhante ao pressuposto para $I 2_{f}$ ocorre a verificação dos indicadores condições de pagamento $\left(I 3_{f}\right)$ e flexibilidade de negociação $\left(I 4_{f}\right)$ propostos pelas Equações (15) e (16),

$$
\begin{aligned}
& \mathrm{I} 3_{\mathrm{f}}=\sum_{0=1}^{\mathrm{n}} \sum_{\mathrm{p}=1}^{\mathrm{n}} \frac{\left(\frac{\mathrm{SC_{p }}}{\mathrm{n}}\right)_{\mathrm{o}}}{\mathrm{n}} \\
& \mathrm{I} 4_{\mathrm{f}}=\sum_{\mathrm{o}=1}^{\mathrm{n}} \sum_{\mathrm{p}=1}^{\mathrm{n}} \frac{\left(\frac{\mathrm{SFp}}{\mathrm{n}}\right)_{\mathrm{o}}}{\mathrm{n}}
\end{aligned}
$$

onde $S C_{p}$ compreende a mensuração da opinião dos profissionais do setor de compras em relação a maneira com que cada fornecedora disponibiliza as suas opções de crédito para as compradoras, forma esta que se prepetua para o fator $S F_{p}$ relativo a disponibilidade de flexibilização com que a negociação é encaminhada, sendo $S C_{p}$ e $S F_{p}$ proporcionais aos valores do fator escala $\alpha$. O desígnio pela coleta somente da opinião deste profissionais parte do pressuposto que somente eles é que realmente podem retornar um feedback real do comportamento do fornecedor em questão para com as condições propostas.

Enquanto isso, o indicador $I 5_{f}$, relativo a situação da empresa perante o seu mercado de atuação, é proposto a partir da visualização da maneira com que a fornecedora está conduzindo as suas ações perante o seu público externo, bem como da sua abrangência, crescimento nos últimos anos de atuação e do nível de insatisfação dos seus clientes, por meio de consultas realizadas a partir da opinião de especialistas da área e de dados coletados na internet, gerando assim a medida do índice $S M_{o}$, por meio da avaliação realizada por um dos profissionais de compras designado pelos gestores da empresa, obtendo-se ao final o cálculo deI5 $5_{f}$ conforme mostra a Equação (17), sendo $S M_{o} \propto$ fator escala $\alpha$.

$$
\mathrm{I} 5_{\mathrm{f}}=\sum_{\mathrm{o}=1}^{\mathrm{n}} \mathrm{SM}_{\mathrm{o}}
$$

Para $\mathrm{o}$ indicador $I 6_{f}$ referentea quantidade proporcional do pedido original atendido pelo fornecedor, descrito pela Equação (19), tem-se por referência o cruzamento entre as descrições detalhadas de cada item que compõe o escopo do pedido $(M B)$ e a disponibilidade da cobertura destes por parte da empresa solicitada $\left(M O_{f}\right)$, resultando no cálculo do índice $\gamma_{f}$, conforme a Equação (18) descrita a seguir,

$$
\begin{gathered}
\gamma_{\mathrm{f}}=\frac{\mathrm{MO}_{\mathrm{f}}}{\mathrm{MB}} * 100 \\
\mathrm{I}_{\mathrm{f}}=\left\{\begin{array}{c}
1 \rightarrow \gamma_{\mathrm{f}} \leq 0,90 \\
2 \rightarrow 0,90<\gamma_{\mathrm{f}}<0,94 \\
3 \rightarrow 0,94 \leq \gamma_{\mathrm{f}} \leq 0,96 \\
4 \rightarrow 0,96<\gamma_{\mathrm{f}}<1,00 \\
5 \rightarrow \gamma_{\mathrm{f}}=1,00
\end{array}\right.
\end{gathered}
$$

onde os valores de cada um dos intervalos estão determinados com base na série histórica das entregas da área de TI concebidos durante o ano de 2012, bem como da experiência dos profissionais da área consultados.

Por fim, os prazos de entrega $\left(I 7_{f}\right)$ e de pagamento $\left(I 8_{f}\right)$ estão fundados de acordo com o tempo necessário para o cumprimento dessas atividades, ondeo primeiro é relativo ao período médio transcorrido desde o fechamento do negócio até a chegada de todos dos produtos na IES $\left(\vartheta_{f}\right)$, enquanto o segundo se trata do período de carência em que a fornecedora trabalha com a instituição $\left(\sigma_{f}\right)$, partindo do pressuposto de quanto maior a diferença entre o período de realização do pedido até o primeiro pagamento, melhor são as condições do fluxo de caixa da empresa. Para tanto, ambas as definições estão propostas conforme as Equações (21) e (23), a partir dos valores de $\vartheta_{f}$ e $\sigma_{f}$, respectivamente, relativos aos valores em unidades temporais encontrados para cada fornecedor, de acordo com as Equações (20) e (22), 


$$
\begin{gathered}
\delta_{\mathrm{f}}=\sum_{\mathrm{o}=1}^{\mathrm{n}} \sum_{\mathrm{it}=1}^{\mathrm{n}} \frac{\left(\frac{\overline{\mathrm{TE}}_{\mathrm{it}}}{\mathrm{n}}\right)_{\mathrm{o}}}{\mathrm{n}} \\
\mathrm{I}_{\mathrm{f}}=\left\{\begin{array}{c}
1 \rightarrow \vartheta_{\mathrm{f}} \leq 7 \\
2 \rightarrow 7<\vartheta_{\mathrm{f}}<15 \\
3 \rightarrow 15 \leq \vartheta_{\mathrm{f}} \leq 22 \\
4 \rightarrow 22<\vartheta_{\mathrm{f}}<30 \\
5 \rightarrow \vartheta_{\mathrm{f}} \geq 30
\end{array}\right. \\
\mathrm{I}_{\mathrm{f}}=\left\{\begin{array}{c}
1 \rightarrow \sigma_{\mathrm{f}} \leq 30 \\
\rho_{\mathrm{f}}=\mathrm{TP}_{\mathrm{f}} \\
3 \rightarrow 30<\sigma_{\mathrm{f}}<45 \\
4 \rightarrow 60<\sigma_{\mathrm{f}} \leq 60 \\
5 \rightarrow \sigma_{\mathrm{f}} \geq 90
\end{array}\right.
\end{gathered}
$$

onde $\overline{T E}$ é o tempo médio para a entrega de cada um dos itens solicitados, it $\in$ $\{1,2, \ldots, n\}$ são os itens no pedido e $T P$ é o prazo de pagamentos estipulado pelo fornecedor, sendo os valores atribuídos para as escalas consolidados de acordo com o nível de exigência proposto por parte dos gestores da empresa.

Com o estabelecimento desses critérios por meio do TCO realizado na IES e das alternativas de fornecimento dos materiais elencadas, parte-se para a etapa de ordenação, utilizando-se para tanto dos conceitos definidos a respeito dos métodos de AHP Referenciada e B-G, obtendo com o cruzamento dos dados de cada fornecedor a melhor opção para se consolidar o pedido dos equipamentos de TI estimados anteriormente, chegando aos resultados estabelecidos conforme mostra a Tabela 4 a

\begin{tabular}{|c|c|c|c|c|c|c|c|c|c|c|c|c|}
\hline \multirow{2}{*}{$f$} & \multicolumn{8}{|c|}{$I u_{f}$} & \multirow{2}{*}{$\begin{array}{l}w_{f} \\
(\%)\end{array}$} & \multirow{2}{*}{$\begin{array}{c}\mu_{f} \\
(\%)\end{array}$} & \multirow{2}{*}{$\begin{array}{c}\Delta_{f} \\
(\%)\end{array}$} & \multirow{2}{*}{$\begin{array}{c}\rho_{f} \\
(\%)\end{array}$} \\
\hline & 1 & 2 & 3 & & 5 & 6 & & 8 & & & & \\
\hline 1 & 4 & 4 & 4 & 4 & 4 & 3 & 2 & 5 & 23,8 & 24,1 & $-0,34$ & 24,0 \\
\hline 2 & 2 & 3 & 3 & 2 & 3 & 5 & 4 & 5 & 21,9 & 22,5 & $-0,65$ & 22,2 \\
\hline 5 & 3 & 4 & 4 & 4 & 4 & 1 & 3 & 2 & 19,2 & 19,2 & 0,04 & 19,3 \\
\hline
\end{tabular}
seguir,

Tabela 4 - Resultados encontrados para a priorização proposta

\begin{tabular}{|l|l|l|l|l|l|l|l|l|l|l|l|l|}
\hline $\mathbf{4}$ & 1 & 3 & 3 & 4 & 4 & 1 & 4 & 3 & 17,6 & 16,9 & 0,69 & 17,3 \\
\hline $\mathbf{3}$ & 2 & 2 & 3 & 3 & 2 & 2 & 3 & 5 & 17,3 & 17,1 & 0,25 & 17,2 \\
\hline
\end{tabular}

Para tanto, através da utilização destes dois modelos foi possível definir que a melhor escolha dentre as cinco disponibilizadas no estudo é a denominada por $f=1$,equivalente a $24 \%$ do resultado total, sendo superior as outrastanto para o levantamento Referenciado $\left(w_{1}=\right.$ $23,82 \%)$ quanto para o B-G $\left(\mu_{1}=\right.$ $24,16 \%$ ), consequência de uma série de bons resultados detectados para todos os indicadores, com exceção aos valores obtidos em $f=6$ (3) e $f=7$ (2).

No que tange os resultados obtidos para asalternativas, cabe destacar os bons resultados obtidos por $f=2$ quanto a questão do cumprimento com fidelidade dos pedidos proposto para orçamento $\left(I 6_{2}=5\right)$, bem como de $f=5$ para os indicadores $I 2_{5}$ até $I 5_{5}$ (4), relativos as questões qualitativas no processo de compra. Nota-se também que a diferença entre os resultados propostos pelas metodologias AHP distorcem de maneira superior em $f=4(0,69 \%)$, o que pode ser considerado um valor extremamente pequeno se comparado proporcionalmente ao valor obtido no resultado médio da alternativa em questão.

Por fim é proposto o gerenciamento das informações por meio de um banco de dados, partindo-se do cadastro dos dados mencionados durante o desenvolvimento do trabalho para cada um dos fornecedores já contactados pela IES, estando estes vinculados em uma planilha eletrônica simples, pois a demanda de informações a respeito do tema em um horizonte decurto/médio prazo não é considerada de grande relevância para a implantação de algum software específico para a consolidação de tal função.

\section{CONCLUSÃO}

Diante dos fatos expostos neste artigo, é possível afirmar que o objetivo de apoiar a 
decisão no momento da compra de equipamentos de informáticaatravés da utilização dos conceitos a respeito do Custo Total de Propriedade e do Processo de Análise Hierárquica, para uma Instituição de Ensino Superior privada localizada na região central do Estado do Rio Grande do Sul, foi cumprido de maneira satisfatória pelos pesquisadores, retornando aos interessados no assunto uma visão mais real, sistêmica e dinâmica dos possíveis caminhos ótimos para esse tipo de atividade a serem seguidos com a aplicação desses procedimentos para o caso em questão. Como pontos complementares, cabe destacar a maneira prática e dinâmica com que ambos os métodos se adaptaram para esse fim, tornando por consequência o trabalho de quem organiza esse tipo de projeto mais simples, padronizado e dinâmico, retornando respostas rápidas sobre o comportamento dos investimentos futuros a serem realizados.

Quanto ao estudo dos processos de compra ou solicitação de serviços para TI, pode-se a partir da situação atual em que estas ocorrem, se propor uma nova metodologia que contempla um estudo mais amplo sobre os dados qualitativos de cada fornecedor antes da solicitação, bem como da elaboração, após a aquisição, de relatórios contendo o nível obtido de custobenefício da negociação, gerando assim um amplo histórico a respeito de cada um dos fornecedores, guiando a instituição em futuras solicitações.

Quanto às limitações da pesquisa, é possível citar a falta de uma metodologia pré-estabelecida para a adoçãomais organizada do TCO, bem como de aplicações que envolvam um âmbito mais científico de análise dos problemas, o que pode tornar a aplicação desconexa com a realidade estudada. Como recomendação para trabalhos futuros, sugere-se a aplicação desta não somente para o caso de projetos específicos de TI, e sim abranger todas as formas de aquisição de materiais existentes na IES estudada, além da aplicação em casos que abordem projetos em outros tipos de organizações privadas e públicas.

\section{REFERÊNCIAS}

ALMEIDA, K.D.M., BORGES, M.F., CARVALHO, C.V.A. \& LIMA JUNIOR, J.A.T.2010. Custo total de propriedade aplicado ao centro de documentação histórica da Universidade Severino Sombra: um estudo de caso. Revista TECCEN,3, 1-21.

BANDEIRA, A.A. 2009. Indicadores de desempenho: instrumento à produtividade organizacional. 1 ed. Rio de Janeiro,Qualitymark.

BELTON, V.A.\& GEAR, T. 1985.The legitimacy of rank reversal.Omega, 13, 143 $-144$.

COUSINS, P. D., LAMMING, R. C., LAWSON, B. \&SQUIRE, B.2008.Strategic Supply Management. Harlow, Pearson Education.

DEGRAEVE, Z., ROODHOOFT, F. E. \&VAN DOVEREN, B.2005.The use of total cost of ownership for strategic procurement: a company-wide management information system.Journal of the Operational Research Society,56, 5159.

DRUCKER, P. F. \&MARCIARIELLO J. A. 2005.The Effective Executive in Action. New York, Harper Business.

ELLRAM, L.M.\& ZSIDISIN, G.2002.Factor that drive purchasing and supply management's use of information technology.IEE Transaction on Engineering Management,49, 269-281.

FEIMAN, J. 2012. Application TCO Model: $\quad$ Categories and Architectures.Disponível em: 
$<$ http://www.gartner.com>Acesso

em $15 / 09 / 2012$.

GOMES, L.F.A.M., ARAYA, M.C. G. \& CARIGNANO, C. 2011. Tomada de decisões em cenários complexos: Introdução aos métodos discretos de apoio multicritério à decisão. São Paulo, Cengage Learning.

GOMES, C.F.\& GOMES, L.F.A.M. 2012. Tomada de decisão gerencial: Enfoque Multicritério. São Paulo, Atlas.

GUITOUNI, A. \& MARTEL, J.M. Tentative guidelines to help choosing an appropriate MCDAmethod.1998.European Journal of Operational Research, 109,501 $-521$.

HANSEN, G.L. 2001.Limites e Potenciais das Novas Modalidades de Gestão Universitária. São Paulo, Editores Associados.

INEP - Instituto Nacional De Estudos E Pesquisas Educacionais Anísio Teixeira. Sinopse da Educação Superior. Disponível em:

$<$ http://www.inep.gov.br/superior/censosup erior/sinopse/>. Acesso em: 15/09/2012.

KAPLAN, R. S. \& NORTON, D.P. 2008.A Execução Premium. Rio de Janeiro, Campus.

KOSIER, J.M. \& STRONG, D. 2006.Supply/demand chain modeling utilizing logistical-based costing. Journal of Enterprise Information Management, 19,346-360.

LOBATO, D. M., FILHO, J. M., TORRES, M. C. S. \& RODRIGUES, M. R. A. 2009.Estratégias de empresas. Rio de Janeiro, FGV.

LIMA FILHO, R. N.,BRUNI, A. L., SAMPAIO, M. S. \& PEREIRA, A.G.2011. Conceitos relevantes de custos: A visão de textos didáticos, o olhar da Teoria da Contabilidade e a percepção de discentes.ABCustos Associação Brasileira de Custos, 6.

LOOTSMA, F.A. Scale sensitive in the multiplicative AHP and Smart. 1993. Journal of Multi-Criteria Decision Analysis, 2,87 - 110.

MENEGAT, V.2006. Utilização dos sistemas de custos pelas Instituições de Ensino Superior do Sistema ACAFE - SC. 6 Congresso USP de Controladoria $e$ Contabilidade. USP - São Paulo.

MERLO, R. A. \& BEUREN, I.M.Sistema de informações para a gestão econômicofinanceira de uma universidade comunitária: um estudo de caso da UNOESC. In: BEUREN, I. M. 2004. Sistema de informações para a gestão universitária. São Miguel do Oeste, UNOESC.

MINAYO, M.C.S.2010. Pesquisa Social: teoria, método e criatividade.Petrópolis, Vozes.

PORTER, M. 2009. Competitividade. São Paulo, Campus.

RAMOS, M.M. 2004.Interaction between management accounting and supply chain management.Supply Chain Management: An international Journal, 9, 134-138.

SAATY, T.L. 1980. The Analytic Hierarchy Process.New York, McGraw Hill.

SAATY, T.L. Decision making with the Analytic Hierarchy Process. 2008.International Journal of Services Sciences, 1, 83 - 98.

SAATY, T. L. \& VARGAS, L.G. 2012.Methods, concepts \& applications of the Hierarchy Process.New York, Springer. 
SALIBA, F. M. P.2006. A adoção do custo total de propriedade no processo de compras de grandes empresas brasileiras: Um estudo de caso. Dissertação, Universidade Federal do Rio de Janeiro.

SANTOS, S.M. S., BORNIA, A. C. \&LEITE, M.S.A.2010. Sistema de Gestão de Custos Associado à Cadeia de Valor. ABCustos Associação Brasileira de Custos,5.

ATSON, S. R. \& FREELING, A.N.S. 1982. Assessing attribute weights by ratios. Omega, 10, 582 - 583.

ZACHARIASSEN F. \&ARLBJØRN J. S. 2011.Exploring a differentiated approach to total cost of ownership.Industrial Management \& Data Systems, 111, 448469. 